\title{
Prevalence and Incidence of Pharyngeal Gonorrhea in a Longitudinal Sample of Men Who Have Sex with Men: The EXPLORE Study
}

\author{
Sheldon R. Morris, ${ }^{1}$ Jeffrey D. Klausner, ${ }^{2}$ Susan P. Buchbinder, ${ }^{2}$ Sarah L. Wheeler, ${ }^{2}$ Beryl Koblin, ${ }^{4}$ Thomas Coates, ${ }^{3}$ \\ Margaret Chesney, ${ }^{5}$ and Grant N. Colfax ${ }^{2}$ \\ 'Department of Community and Family Medicine, University of California San Francisco, and ${ }^{2}$ San Francisco Department of Public Health, \\ San Francisco, and ${ }^{3}$ University of California, Los Angeles, California; ${ }^{4}$ New York Blood Center, New York, New York; and ${ }^{5}$ National Center \\ of Complementary and Alternative Medicine, Bethesda, Maryland
}

Background. The prevalence of gonorrhea of the pharynx among select samples of men who have sex with men (MSM) ranges from $9 \%$ to $15 \%$. To our knowledge, there have been no longitudinal studies in a prospective MSM cohort to estimate pharyngeal gonorrhea incidence or predictors of infection. We examined the prevalence, incidence, and sociodemographic and behavioral predictors of pharyngeal gonorrhea in a cohort of sexually active, human immunodeficiency virus-negative MSM.

Methods. We conducted a prospective study of pharyngeal gonorrhea among MSM who were enrolled in a behavioral intervention study to prevent human immunodeficiency virus infection (Project EXPLORE). Participants were enrolled in this ancillary study from March 2001 through July 2003. At baseline and every 6 months thereafter until 31 July 2003, participants were tested for pharyngeal gonorrhea and were administered a questionnaire regarding their oral sex practices. Rectal and urethral gonorrhea testing were also performed.

Results. Prevalence of pharyngeal gonorrhea was 5.5\% (136 cases diagnosed from 2475 tests). The incidence rate was 11.2 cases per 100 person-years. Pharyngeal gonorrhea was positively associated with younger age and the number of insertive oral sex partners in the past 3 months. Ejaculation did not increase the risk of pharyngeal gonorrhea. Gonorrhea of the pharynx was asymptomatic in $92 \%$ of cases.

Conclusions. The pharynx is a common, asymptomatic reservoir for gonorrhea in sexually active MSM.

In the 2003 Centers for Disease Control and Prevention Sexually Transmitted Disease (STD) Surveillance Report, men who have sex with men (MSM) presenting to STD clinics in 2003 had a median prevalence of $15.3 \%$ for gonorrhea at any anatomic site [1]. The pharynx is the most common site of gonococcal infection among MSM [2-6]. Rates of pharyngeal gonorrhea among MSM tested in HIV clinics, STD clinics, anonymous testing sites, and bathhouses range from $3 \%$ to 15\% [7-9]. In San Francisco, where more than onehalf of reported cases of gonorrhea are attributed to MSM, the pharynx accounts for an increasing number

Received 1 May 2006; accepted 31 July 2006; electronically published 10 October 2006

Reprints or correspondence: Dr. Grant Colfax, HIV/AIDS Statistics, Epidemiology and Intervention Research Section, San Francisco Dept. of Public Health, 25 Van Ness Ave., Ste. 710, San Francisco, CA 94102 (grant.colfax@sfdph.org).

Clinical Infectious Diseases 2006; 43:1284-9

(C) 2006 by the Infectious Diseases Society of America. All rights reserved. $1058-4838 / 2006 / 4310-0009 \$ 15.00$ of total gonorrhea cases among this population, possibly because of increased screening [10].

Pharyngeal gonorrhea may be self limiting, with up to $50 \%$ of cases detected by culture spontaneously remitting within a week and all cases spontaneously remitting within 3 months $[11,12]$. This, however, does not eliminate the importance of pharyngeal gonorrhea as a source of genital infection, because oral sex has been shown to be an independent risk factor for urethral gonorrhea and, in some cases, may be the only identified risk factor for infection $[6,13,14]$.

Whereas previous research provides only cross-sectional data on pharyngeal gonorrhea, this longitudinal cohort study enabled us to measure both prevalence and incidence of pharyngeal gonorrhea among sexually active, HIV-negative MSM, and to compare rectal and urethral gonorrhea incidence rates with pharyngeal gonorrhea rates during the same period. We also determined the behavioral, clinical, and sociodemographic predictors of pharyngeal gonorrhea infection. 


\section{METHODS}

The EXPLORE study was a multicity, randomized, controlled trial of a behavioral intervention for the prevention of HIV infection among MSM. From January 1999 to February 2001, a total of 736 MSM from San Francisco were enrolled in the study. Recruitment occurred through outreach in neighborhoods with significant populations of MSM; other venues frequented by MSM, including bathhouses and health and sex clubs; STD clinics; with advertisements; and through referrals from other participants. Eligibility requirements included being HIV antibody-negative at baseline, being at least 18 years of age, reporting anal sex in the past year with at least 1 man, and not being currently in a mutually monogamous relationship for $\geqslant 2$ years with a known HIV antibody-negative man. Eligible men were randomized to the intervention (10 counseling sessions delivered over a 6-month period, followed by quarterly booster sessions with HIV testing and counseling every 6 months) or to the control arm (standard HIV testing and counseling every 6 months). All San Francisco study visits occurred at the AIDS Office of the San Francisco Department of Public Health. Details on the EXPLORE study have been published elsewhere [15].

As part of the EXPLORE study, willing participants were tested for rectal and urethral gonorrhea. Beginning 12 March 2001, San Francisco EXPLORE participants were offered enrollment into a pharyngeal gonorrhea ancillary study that was approved by the Institutional Review Board of the University of California, San Francisco. Informed consent was obtained from all participants. At each semiannual EXPLORE visit, a pharyngeal swab was collected and participants completed a self-administered paper questionnaire that asked about recent oral sex behavior, including number of insertive (i.e., the participant had a sex partner's penis in his mouth) and receptive (i.e., the participant put his penis in a sex partner's mouth) partners and episodes, by HIV status and ejaculation. Participants were also asked whether they had a sore throat at the visit and the number of sore throats they had experienced in the past 3 months that had lasted at least 1 day. Because pharyngeal gonorrhea can spontaneously resolve after 3 months, we asked only about behaviors and throat symptoms occurring during the 3 months immediately prior to the study visit, rather than during the entire 6-month interval between study visits.

Participants were tested for pharyngeal gonorrhea, regardless of symptoms. From 12 March 2001 to 15 January 2002, pharyngeal samples were tested using ligase chain reaction (Abbott Laboratories), which has a sensitivity of $94.7 \%$ and a specificity of $97.8 \%$ for pharyngeal gonorrhea [3]. Because of the discontinuation of the ligase chain reaction test, pharyngeal samples collected after 15 January 2002 were tested using strand displacement amplification (BD Laboratories), which has a sensitivity of $87.5 \%$ and a specificity of $96.1 \%$ for pharyngeal gonorrhea [16]. The strand displacement amplification testing method for gonorrhea was initiated on 21 March 2002 for rectal samples and on 4 February 2002 for urethral samples; all participants were tested, regardless of symptoms. All men who had positive test results for gonorrhea were offered treatment with a single $400 \mathrm{mg}$ dose of oral cefixime, and were provided treatment, as well as STD clinic referrals for their current regular sex partners.

Statistical analyses were performed with SAS software, version 9.1 (SAS Institute). Prevalence and incidence calculations conservatively excluded pharyngeal results obtained from the same individual within 45 days to avoid counting either residual gonococcal material from old cases or treatment failures as new cases. Using the previously reported estimate that up to $50 \%$ of pharyngeal gonorrhea cases may spontaneously remit within 1 week [12], we estimated that $<1$ person out of 100 would continue to be infected with persistent pharyngeal gonorrhea after 45 days. The point prevalence of pharyngeal gonorrhea over 3-month calendar intervals was calculated using the total number of positive pharyngeal gonorrhea tests divided by the total number of pharyngeal tests performed. The incidence rate was calculated as the number of cases of pharyngeal gonorrhea per 100 person-years of observation. Positive cases at the baseline pharyngeal testing visit were treated but excluded from the incidence analysis, such that all follow-up testing would relate to a documented negative status. Person-years of observation was defined as the period from the date of the first test result through the date of the last test result.

Univariate and multivariate modeling for predictors of pharyngeal gonorrhea used General Estimating Equation regression to account for repeated measures in the same individuals. To be included in the multivariate model, it was required that variables be associated with pharyngeal gonorrhea with a $P$ value $\leqslant .1$ in the univariate model. Continuous variables were divided into ordinal variables by quartiles, because a first-order linear association was not assumable. Participants listed the number of oral sex partners by whether ejaculation occurred, which could possibly allow for 1 partner to be counted twice (if ejaculation occurred in 1 episode and no ejaculation occurred during another episode with the same partner). Therefore, the number of oral sex partners was calculated using the largest number of partners from either the "ejaculation" portion or the "no ejaculation" portion. Because participants most commonly reported nonejaculating partners, our estimates may exclude some partners who had always ejaculated. For the reference group, the first quartile for the number of partners included those that reported 1 partner or no partners in the past 3 months.

\section{RESULTS}

The primary EXPLORE study analysis did not demonstrate a statistically significant reduction in HIV infection rates between study arms; these data and baseline characteristics of EXPLORE 
participants have been previously reported [15, 17]. In all, 603 $(88 \%)$ of the 686 EXPLORE participants who made a semiannual visit after 11 March 2001 enrolled into the ancillary study. Of these participants, 206 (34\%) were recruited from street outreach, 127 (21\%) from print advertisements, 71 (12\%) from clubs or bars, $48(8 \%)$ from other completed research studies, and $40(7 \%)$ were referred by friends. The remaining 111 participants $(18 \%)$ were recruited from a variety of other venues, with no more than $2 \%$ of the total sample recruited from any 1 source, including physician referrals, bathhouses and sex clubs, community-based organizations, the Internet, and community forums.

The mean age of participants was 36 years; $71 \%$ were nonLatino white; $72 \%$ had education beyond high school; and $69 \%$ had an annual income $>\$ 30,000$. Few participants reported receiving a diagnosis of an STD in the 6 months prior to enrollment in the EXPLORE study; however, sexual risk behavior was high, with nearly one-half of participants reporting having unprotected anal sex with an unknown HIV status or an HIVpositive partner, and nearly three-quarters reporting at least 1 episode of sex while drinking or high (table 1).

Participants completed a median of 4 pharyngeal gonorrhea study visits and attended $97 \%$ of all the scheduled semiannual study visits. Of the 603 participants enrolled, a sufficient oral sample was never obtained from 3 people. In 206 (7.6\%) of the 2682 study visits, no pharyngeal sample was collected. In another 69 visits, a result for pharyngeal gonorrhea was received, but the questionnaire was not filled out; and in 4 additional instances, all data were complete except for information on partner HIV infection status. One sample was not included in analysis because of a lab processing error.

Twenty-four participants experienced HIV seroconversion during the study and were censored at the time of their first HIV-positive test. These individuals completed 82 visits. At the first testing visit, $30(5 \%)$ of 600 participants had a positive pharyngeal gonococcal test result. Including the baseline visit, $110(18 \%)$ of 600 participants had at least 1 positive pharyngeal gonorrhea test result at some point during the study. Twentytwo (20\%) of the 110 individuals who had pharyngeal gonorrhea experienced $>1$ case of pharyngeal gonorrhea over the study period, including 2 participants who had 3 positive test results and 1 participant who had 4 positive test results. Five (21\%) of the 24 individuals who became HIV-positive during the study were diagnosed with at least 1 case of pharyngeal gonorrhea.

Prevalence. Pharyngeal gonorrhea prevalence for the whole study was 5.5\% (136 of 2475 pharyngeal tests; $95 \%$ CI, 4.7\%$6.4 \%$ ). Three-month calendar point prevalence of pharyngeal gonorrhea ranged from $2.8 \%$ to $8.0 \%$ during the study (figure 1). From 21 March 2002 (after strand displacement amplification testing had been implemented for all anatomic sites) through 1 August 2003, a total of 89 (6.4\%) of 1401 pharyngeal
Table 1. Baseline characterisitics of study participants.

\begin{tabular}{|c|c|}
\hline Patient characteristic & $\begin{array}{c}\text { No. }(\%) \\
\text { of study } \\
\text { participants } \\
(n=603)\end{array}$ \\
\hline \multicolumn{2}{|l|}{ Age, years } \\
\hline $18-29$ & $159(26)$ \\
\hline $30-44$ & $349(58)$ \\
\hline$\geqslant 45$ & $95(16)$ \\
\hline \multicolumn{2}{|l|}{ Race/ethnicity } \\
\hline Non-Latino white & $426(71)$ \\
\hline African American & $37(6)$ \\
\hline Latino & $90(15)$ \\
\hline Asian/Pacific Islander & $31(5)$ \\
\hline Other & $19(3)$ \\
\hline \multicolumn{2}{|l|}{ Annual Income } \\
\hline$<\$ 30,000$ & $187(31)$ \\
\hline$\$ 30,000-60,000$ & $216(36)$ \\
\hline$>\$ 60,000$ & $200(33)$ \\
\hline \multicolumn{2}{|l|}{ Education } \\
\hline High school or less & $170(28)$ \\
\hline At least some college, but no graduate school & $273(45)$ \\
\hline At least some graduate school & $160(27)$ \\
\hline \multicolumn{2}{|l|}{$\begin{array}{c}\text { Diagnosed with an STD in } 6 \text { months prior to } \\
\text { enrollment }\end{array}$} \\
\hline Gonorrhea & $14(2)$ \\
\hline Urethral & $12(2)$ \\
\hline Rectal & $0(0)$ \\
\hline Pharyngeal & $2(<1)$ \\
\hline Chlamydia & $24(4)$ \\
\hline Syphilis & $0(0)$ \\
\hline \multicolumn{2}{|l|}{ Sexual behavior in 6 months prior to enrollment } \\
\hline Any unprotected anal sex & $392(65)$ \\
\hline $\begin{array}{l}\text { Any unprotected anal sex with an HIV-positive or } \\
\text { unknown partner }\end{array}$ & $272(45)$ \\
\hline Any unprotected receptive oral sex with ejaculation & $247(41)$ \\
\hline Any sex while drinking or high & $447(74)$ \\
\hline
\end{tabular}

NOTE. STD, sexually transmitted disease.

tests had positive results. For the same period, $15(1.8 \%)$ of 851 rectal swabs and $8(0.6 \%)$ of 1374 urethral samples had positive test results for gonorrhea.

Incidence. There were 943.2 person-years of observation. There were 85 persons with at least 1 incident pharyngeal gonorrhea case and there was a total of 106 incident cases. The overall incidence rate (including repeat cases) was 11.2 cases per 100 person-years (95\% CI, 9.3-13.4). Beginning 21 March 2002 (the period during which specimens from all anatomic sites were tested using the same method), the incidence rate for pharyngeal gonorrhea was 11.7 cases per 100 person years (95\% CI, 8.8-15.3). In comparison, the incidence rates were 3.5 cases per 100 person-years $(95 \% \mathrm{CI}, 1.6-7.0)$ for rectal gonorrhea and 1.5 cases per 100 person years (95\% CI, 0.63.4) for urethral gonorrhea.

Tests outside of the study. At each semiannual EXPLORE visit, participants were asked if they had received a diagnosis of 


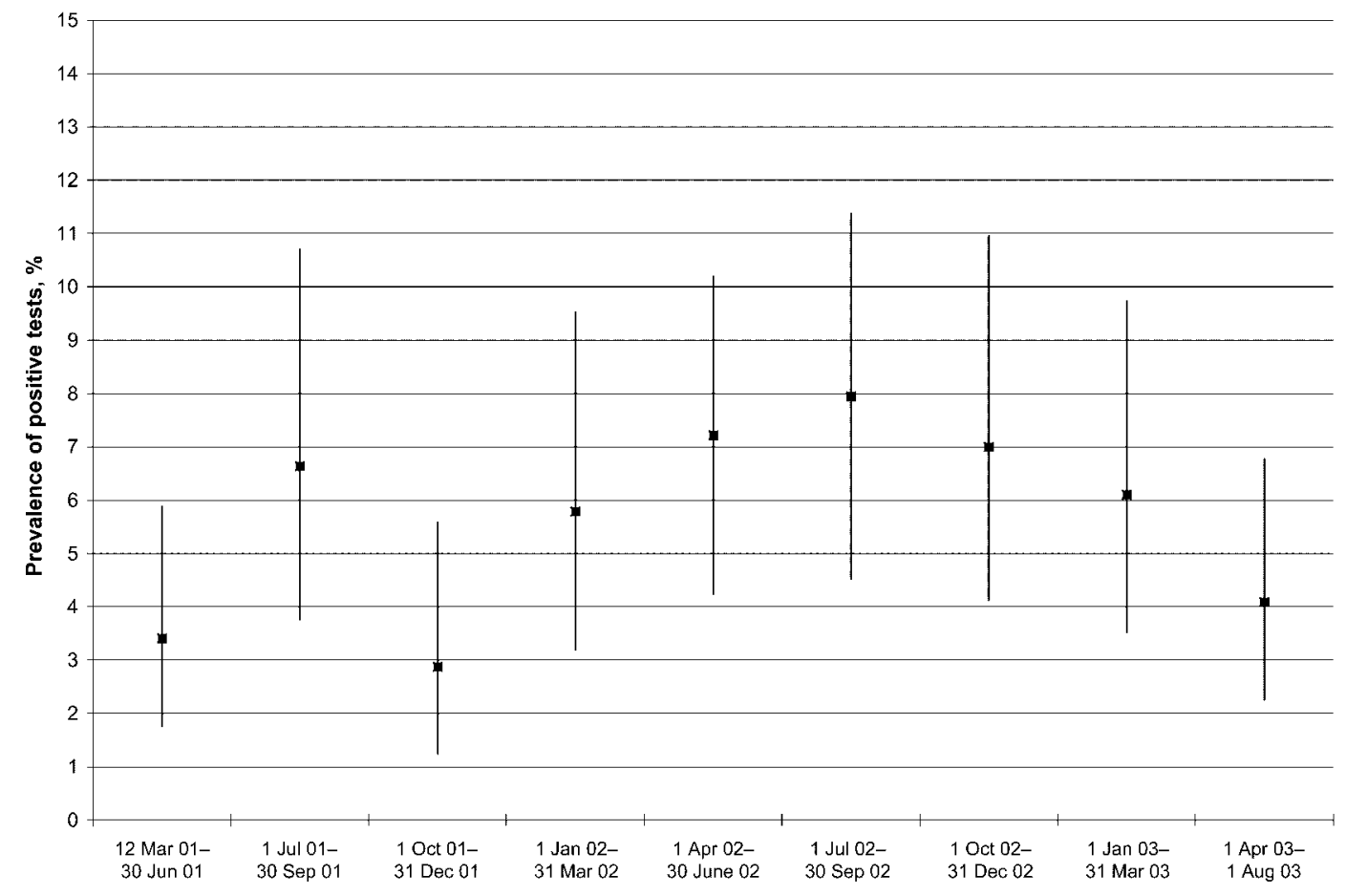

Figure 1. Point prevalence of pharyngeal gonorrhea in the San Francisco EXPLORE Cohort of HIV-negative men who have sex with men, from March 2001 through July 2003. Bars, 95\% Cls.

or treatment for gonorrhea from a medical provider since the last study visit. During the entire study period, there were 2696 semiannual visits among 603 individuals. There were 39 reports (1.5\%) of outside pharyngeal gonorrhea, $19(0.7 \%)$ of rectal gonorrhea, and 44 (1.6\%) of urethral gonorrhea. From 21 March 2002 to 1 August 2003 (the period during which specimens from all anatomic sites were tested using the same method), a total of 580 individuals had 1524 semiannual visits. There were 23 reports (1.5\%) of outside pharyngeal gonorrhea, $14(0.9 \%)$ of rectal gonorrhea, and 23 (1.5\%) of urethral gonorrhea.

Predictors of pharyngeal gonorrhea. In univariate analysis, a pharyngeal gonorrhea diagnosis was associated with younger age and having more partners in the previous 3 months (table 2). African American men had reduced odds of pharyngeal gonorrhea, compared with those identifying as non-Latino white. Having at least $1 \mathrm{HIV}$-positive insertive oral sex partner was also associated with pharyngeal gonorrhea. There was no difference in pharyngeal gonorrhea rates by EXPLORE study arm. As the receptive oral sex partner, not allowing ejaculation in the mouth was protective, compared with allowing ejaculation in the mouth, but it was of borderline statistical significance.

Of participants who reported current sore throat symptoms, $5.1 \%$ had pharyngeal gonorrhea, compared with $5.6 \%$ of those who did not report a sore throat at the time of the visit $(P$ $=.9$ ). The number of sore throats in the 3 months preceding each visit also had no consistent association with a positive pharyngeal gonorrhea test.

In multivariate analysis, the remaining significant positive predictors $(P \leqslant .05)$ of pharyngeal gonorrhea were younger age and an increased number of insertive oral sex partners. African American race remained a negative predictor of pharyngeal gonorrhea. In exploratory analyses, we found no significant demographic or risk behavior predictors in comparing persons with repeat cases of pharyngeal gonnorhea with those with only 1 case of pharyngeal gonorrhea reported during the study period.

\section{DISCUSSION}

This study indicates that pharyngeal gonorrhea is common among sexually active, HIV-negative MSM and may, therefore, serve as an important reservoir for infection at genital sites. Pharyngeal gonorrhea was most often asymptomatic, with no significant relationship with sore throat symptoms. Pharyngeal gonorrhea was far more prevalent than rectal or urerthral gonorrhea. Although this finding might be expected, because participants may have been more likely to have rectal or urethral symptoms and, therefore, seek care, very few participants reported receiving a diagnosis of gonorrhea outside of the study. Our findings support previous studies that have found the pharynx to be the most common-and often the only-site of gonorrhea infection among MSM [2]. Pharyngeal gonorrhea was 
Table 2. Predictors of pharyngeal gonorrhea.

\begin{tabular}{|c|c|c|c|}
\hline Predictors and behaviors reported in the past 3 months & $\begin{array}{l}\text { Positive pharyngeal } \\
\text { gonorrhea test results }\end{array}$ & Univariate OR (95\% Cl) & $\begin{array}{c}\text { Multivariate } \\
\text { adjusted OR } \\
(95 \% \mathrm{Cl})^{\mathrm{b}}\end{array}$ \\
\hline Insertive, HIV-positive oral sex partner & $45 / 578(7.8)$ & $1.8(1.2-2.7)$ & $1.3(0.8-2.1)$ \\
\hline Allowed no ejaculation in mouth & 72/1478 (4.9) & $0.7(0.5-1.0)$ & $0.8(0.6-1.2)$ \\
\hline \multicolumn{4}{|l|}{ Age, years } \\
\hline $18-29$ & $46 / 629(7.3)$ & $2.2(1.1-4.4)$ & $2.5(1.2-5.0)$ \\
\hline $30-44$ & $75 / 1440(5.2)$ & $1.6(0.8-3.1)$ & $2.0(1.0-4.0)$ \\
\hline$\geqslant 45$ & $15 / 406(3.7)$ & Referent & Referent \\
\hline \multicolumn{4}{|l|}{ Race/ethnicity } \\
\hline Non-Latino white & $103 / 1780(5.8)$ & Referent & Referent \\
\hline African American & 2/149 (1.3) & $0.2(0.1-0.9)$ & $0.2(0.1-0.9)$ \\
\hline Latino & $22 / 349(6.3)$ & $1.1(0.6-2.0)$ & $1.2(0.7-2.2)$ \\
\hline Asian/Pacific Islander & $8 / 126(6.4)$ & $1.0(0.4-2.1)$ & $1.0(0.4-2.3)$ \\
\hline Other & $1 / 71(1.4)$ & $0.2(0.0-1.5)$ & $0.3(0.0-2.2)$ \\
\hline \multicolumn{4}{|l|}{ Annual income } \\
\hline$<\$ 30,000$ & $35 / 741(4.7)$ & $0.8(0.5-1.4)$ & $\ldots^{c}$ \\
\hline$\$ 30,000-\$ 60,000$ & $51 / 892(5.7)$ & $1.0(0.6-1.5)$ & $\ldots^{c}$ \\
\hline$>\$ 60,000$ & $50 / 842(6.0)$ & Referent &..$^{c}$ \\
\hline \multicolumn{4}{|l|}{ Education } \\
\hline High school or less & $40 / 665(6.0)$ & $1.1(0.7-1.9)$ & $\ldots{ }^{c}$ \\
\hline At least some college, but no graduate school & $58 / 1123(5.2)$ & $0.9(0.6-1.5)$ & $\ldots^{c}$ \\
\hline At least some graduate school & $38 / 687(5.5)$ & Referent & $\ldots^{c}$ \\
\hline \multicolumn{4}{|l|}{ Study arm } \\
\hline Standard (referent) & $64 / 1270(5.0)$ & $\ldots$ & $\ldots$ \\
\hline Intervention & $72 / 1205(6.0)$ & $1.2(0.8-1.7)$ & ${ }^{c}$ \\
\hline \multicolumn{4}{|l|}{ No. of insertive oral sex partners in the past 3 months $^{d}$} \\
\hline 0 or 1 & 23/731 (3.2) & Referent & Referent \\
\hline $2-4$ & 24/705 (3.4) & $1.2(0.6-2.4)$ & $1.0(0.5-2.1)$ \\
\hline $5-8$ & $36 / 441(8.2)$ & $3.7(1.9-7.2)$ & $3.1(1.5-6.2)$ \\
\hline$\geqslant 9$ & $50 / 529(9.5)$ & $3.8(2.0-7.3)$ & $3.1(1.6-6.2)$ \\
\hline
\end{tabular}

NOTE. Data are adjusted for repeated measures.

a Data are no. of positive test results/no. of samples tested (\%). A total of 2475 tests for pharyngeal gonorrhea were performed in the study.

b $n=2402$. Seventy-three visits from the univariate analysis were excluded because of missing variables.

c Eliminated in model if the value did not meet the $P<.1$ criterion for inclusion in the multivariate model.

d Insertive oral sex is defined as the insertion of a partner's penis in the participant's mouth.

more common among younger MSM and, as might be expected, had a strong association with having more insertive oral sex partners. Importantly, in multivariate analysis, we found no association of ejaculation during insertive oral sex with pharyngeal gonorrhea. While this result is consistent with no protective effect by not receiving ejaculate, it remains to be reproduced by other studies. This finding does have important implications for MSM who may attempt to reduce their risk of pharyngeal gonorrhea while having insertive oral sex by avoiding exposure to ejaculate. Our finding that pharyngeal gonorrhea was less common among African American MSM was unexpected and may be the result of partner mixing patterns or differences in sexual risk behavior among our African American participants that were not controlled for in our analysis. However, given the relatively small number of African Americans in this study and the borderline significance of the multivariate results, these data should be interpreted with caution.

Our data support the contention that pharyngeal gonorrhea is not usually detected in MSM during routine clinical care. In contrast to urethral and rectal sites, the number of pharyngeal gonorrhea cases reported outside of the study was small in relation to those detected at study visits. This may be explained by the asymptomatic nature of pharyngeal infection and by clinicians being unaware of the need for gonococcal screening of this site.

This study has limitations. The population consisted of sexually active, HIV-negative MSM who were enrolled in a behavioral intervention study in San Francisco, and is, therefore, not representative of the entire population of MSM; risk behaviors and pharyngeal gonorrhea incidence may be different in other populations of MSM. Furthermore, although the EXPLORE intervention showed no significant effect on HIV infection or pharyngeal gonorrhea incidence, it is possible that participation in the study influenced oral sexual behavior in both arms. We also did not have specimens for $7.6 \%$ of study visits; whereas most of these missing samples likely resulted 
from the study counselor forgetting to collect samples or participant refusal, it is possible that, if these specimens had been collected, they could have affected study results. It is also possible that some of our results may have been false positives, especially for rectal and urethral sites where prevalence was low and the positive predictive value of the test may, therefore, also be low. Because we used ligase chain reaction and strand displacement amplification testing to detect cases, our results may not be comparable with studies that have used culture to detect pharyngeal gonorrhea. Another limitation is that multiple cases of pharyngeal gonorrhea occurring within the same individual could have represented persistent infection, because it is recognized that treatment of pharyngeal gonorrhea is not as successful as it is at other sites [12, 18, 19]. However, considering the spontaneous resolution rate of pharyngeal gonorrhea and the fact that cases were not counted as new cases if they occurred $<45$ days apart, potential treatment failures are unlikely to have had much impact on the incidence estimate, and our estimates may actually undercount all pharyngeal gonorrhea cases experienced by the cohort. Finally, the change in testing from using ligase chain reaction to using strand displacement amplification to test samples may have influenced our incidence estimates, although the test characteristics of these 2 techniques are similar.

Our findings highlight the need for routine screening for pharyngeal gonorrhea among sexually active MSM, and they support the 2002 Centers for Disease Control and Prevention STD guidelines. Their recommendations are to screen for gonorrhea in the pharynx at least annually for any sexually active MSM, and every 3-6 months for high-risk MSM (defined as MSM who have multiple, anonymous partners or who have sex in conjunction with illicit drug use) if there is a history of oral-genital exposure [20]. Future studies should include determining the optimal intervals for pharyngeal gonorrhea screening and the degree to which screening and treatment of this mostly asymptomatic infection influences genital and rectal gonorrhea rates among MSM.

\section{Acknowledgments}

Financial support. The EXPLORE trial was supported by the HIV Network for Prevention Trials and was sponsored by the US National Institute of Allergy and Infectious Diseases, the National Institute on Alcohol Abuse and Alcoholism of the National Institutes of Health, Department of Health and Human Services (N01 AI35176, with Abt Associates), the Office of AIDS Research of the National Institutes of Health, US Department of Health and Human Services, through a cooperative agreement with Family Health International (cooperative agreement 5 U01 AI46749) with a subsequent subcontract to Abt Associates, and a cooperative agreement to the University of California, San Francisco (U01 AI47995), the National Institute of Child Health and Human Development, the National Institute on Drug Abuse, and the National Institute of Mental Health. G.N.C.'s work was supported in part by the National Institute on Drug Abuse through a mentored, patient-oriented research (K23) award.

Potential conflicts of interest. All authors: no conflicts.

\section{References}

1. Centers for Disease Control and Prevention (CDC). STD surveillance 2003. Atlanta: CDC, 2003.

2. Kent CK, Chaw JK, Wong W, et al. Prevalence of rectal, urethral, and pharyngeal chlamydia and gonorrhea detected in 2 clinical settings among men who have sex with men: San Francisco, California, 2003. Clin Infect Dis 2005; 41:67-74.

3. Page-Shafer K, Graves A, Kent C, Balls JE, Zapitz VM, Klausner JD. Increased sensitivity of DNA amplification testing for the detection of pharyngeal gonorrhea in men who have sex with men. Clin Infect Dis 2002; 34:173-6.

4. Young H, Manavi K, McMillan A. Evaluation of ligase chain reaction for the non-cultural detection of rectal and pharyngeal gonorrhoea in men who have sex with men. Sex Transm Infect 2003; 79:484-6.

5. Stary A, Ching SF, Teodorowicz L, Lee H. Comparison of ligase chain reaction and culture for detection of Neisseria gonorrhoeae in genital and extragenital specimens. J Clin Microbiol 1997; 35:239-42.

6. Janier M, Lassau F, Casin I, Morel P. Pharyngeal gonorrhoea: the forgotten reservoir. Sex Transm Infect 2003; 79:345.

7. Phipps W, Stanley H, Kohn R, Stansell J, Klausner JD. Syphilis, chlamydia, and gonorrhea screening in HIV-infected patients in primary care, San Francisco, California, 2003. AIDS Patient Care STDS 2005; 19:495-8.

8. Dilley JW, Loeb L, Casey S, Adler B, Rinaldi J, Klausner JD. Treating asymptomatic sexually transmitted diseases at anonymous HIV counseling and testing sites. Sex Transm Dis 2003; 30:874-5.

9. Lister NA, Smith A, Tabrizi S, et al. Screening for Neisseria gonorrhoeae and Chlamydia trachomatis in men who have sex with men at maleonly saunas. Sex Transm Dis 2003; 30:886-9.

10. San Francisco Department of Public Health. San Francisco sexually transmitted disease annual summary, 2003. San Francisco: San Francisco Department of Public Health, 2005.

11. Wallin J, Siegel MS. Pharyngeal Neisseria gonorrhoeae: coloniser or pathogen? Br Med J 1979; 1:1462-3.

12. Hutt DM, Judson FN. Epidemiology and treatment of oropharyngeal gonorrhea. Ann Intern Med 1986; 104:655-8.

13. Marcus $U$, Bremer V, Hamouda $\mathrm{O}$, et al. Understanding recent increases in the incidence of sexually transmitted infections in men having sex with men: changes in risk behavior from risk avoidance to risk reduction. Sex Transm Dis 2006; 33:11-7.

14. Lafferty WE, Hughes JP, Handsfield HH. Sexually transmitted diseases in men who have sex with men: acquisition of gonorrhea and nongonococcal urethritis by fellatio and implications for STD/HIV prevention. Sex Transm Dis 1997; 24:272-8.

15. Koblin B, Chesney M, Coates T. Effects of a behavioural intervention to reduce acquisition of HIV infection among men who have sex with men: the EXPLORE randomised controlled study. Lancet 2004; 364 : $41-50$.

16. Klausner JD, Shayevich C, Moncada J, Moncada SL, Moncada JS. Performance of nucleic acid amplification tests (NAATs) for chlamydia and gonorrhea infections of the oropharynx and rectum. In: Program and abstracts of the 104th Meeting of the American Society of Microbiology (New Orleans, LA). 2004. Available at: http://www.gen-probe .com/pdfs/posters/klausner\%20asm\%202004.pdf.

17. Koblin BA, Chesney MA, Husnik MJ, et al. High-risk behaviors among men who have sex with men in 6 US cities: baseline data from the EXPLORE Study. Am J Public Health 2003; 93:926-32.

18. Moran JS. Treating uncomplicated Neisseria gonorrhoeae infections: is the anatomic site of infection important? Sex Transm Dis 1995;22: $39-47$.

19. Manavi K, Young H, McMillan A. The outcome of oropharyngeal gonorrhoea treatment with different regimens. Int J STD AIDS 2005; 16 : 68-70.

20. Centers for Disease Control and Prevention. Sexually transmitted diseases treatment guidelines 2002. MMWR Recomm Rep 2002; 51(RR6):1-78. 